\title{
A REVIEW ON VEHICLE AGENT
}

\author{
Arpita Mishra \\ Department of Computer Science \\ PSIT, Kanpur, India
}

\author{
Ayush Mohan Awasthi \\ Department of Computer Science \\ PSIT, Kanpur, India
}

\author{
Aryaveer Bajpai \\ Department of Computer Science \\ PSIT, Kanpur, India
}

\begin{abstract}
An Augmented Reality Application for smartphones to guide people in ordinary maintenance of their vehicles anytime, anywhere. This application can bring an immersive experience to the people and can reduce the expenses of users for minor issues. We have built a chat bot module that understands the problem faced by the user and tells the respective solution. If the problem can be fixed by the user himself, the application will respond with the solution otherwise it will ask the user to contact a vehicle mechanic. It includes responses for almost all the warnings such as smoke, strange noise from car, key related problems etc. You can either write your question or simply hold down the button to speak you question.
\end{abstract}

Keywords - Augmented Reality, Unity Engine, Android, Automotive, Inspection, Vuforia, Maintenance, Oil and Gas.

\section{INTRODUCTION}

Car engines and equipment can be a totally unknown world for many of us. Even if you have been driving a vehicle since a long time, you might not be aware of the location of the most relevant parts. So, we often take our vehicle to a mechanic even for minor repairs, due to lack of knowledge and guidance Hence, we designed an Android Application that guides a user to self-troubleshoot and be able to contextually access the instructions required to maintain their car, as and when needed.

\section{TECHNOLOGIES USED}

\section{A. Unity Engine -}

Unity is a cross platform game engine developed by Unity Technologies, first released in June 2005 at Apple Inc's Worldwide developers Conference as Mac OS X-exclusive game engine. The engine can be used for three-dimensional, two-dimensional, virtual reality and augmented reality games.

\section{B. Blender -}

Blender is a free and open source $3 \mathrm{~d}$ computer graphics software tool set used for creating animated films, visual effects,art,3D printed models, motion graphics, interactive $3 \mathrm{~d}$ applications, and computer games. Blender's features include 3D modelling, UV unwrapping, texturing, raster graphic editing, rigging and skinning, fluid and smoke simulation, animating, match moving, video editing and compositing.

\section{Augmented Reality}

It is an interactive experience of a real-world environment where the objects that reside in the real world are enhanced by computer generated perceptual information, sometimes across multiple sensory modalities, including visual, auditory, haptic. AR can be defined as a system that fulfills three basic feature : a combination of real and virtual worlds, real-time interaction, and accurate 3D registration of virtual and real objects

\section{Android Studio}

Android Studio is the official integrated development environment for Google's Android operating system, built on JetBrains, IntelliJ IDEA software and designed specifically for Android Development.Programming languages supported by Android Studio are Java, C++, Kotlin etc.

\section{E. Vuforia}

Vuforia is an augmented reality software development kit for mobile devices that enables the creation of augmented reality applications. It uses computer vision technology to recognize and track planar images and $3 \mathrm{~d}$ objects in real time. This image registration capability enables developers to position and orient virtual objects, such as $3 \mathrm{~d}$ models, in relation to real world objects when they are viewed through the camera of a mobile device. The virtual object then tracks the position and orientation of the image in real time so that the viewers perspective on the objects correspond with the perspective on the target. It thus appears that the virtual object is a part of the real-world scene.

\section{REQUIRED HARDWARE AND SOFTWARE}

- Minimum of $1 \mathrm{~GB}$ of device ram.

- $\quad$ Android OS (Min 5.0 Lollipop) 


\section{International Journal of Engineering Applied Sciences and Technology, 2020 \\ Vol. 5, Issue 7, ISSN No. 2455-2143, Pages 262-264 \\ Published Online November 2020 in IJEAST (http://www.ijeast.com)}

- Access to Device Camera

- Internet Connection

\section{PROBLEMS FACED BY USER}

We face problems in our vehicles time to time and we visit the mechanic before the scheduled service date due to the minor problems that occur in our day-to-day life. Despite the problem being minor, we fail to fix it and pay the huge bills to the mechanic. This problem is serious for people living in areas where vehicle repairing garage is not easily available as people need to travel long distance for small fixes.

\section{PROPOSED SOLUTION}

This involves the use of various technologies. We have used Unity engine to create virtual scenes and animations of 3D models. We made 3D models (.obj) using Blender software. Our application provides an AR based guide for self- trouble shooting the following problems:

- Change Car Battery

- Change Engine Oil

- Change Spark Plug

- Change Wheel of a Car

\section{Battery Change Module}

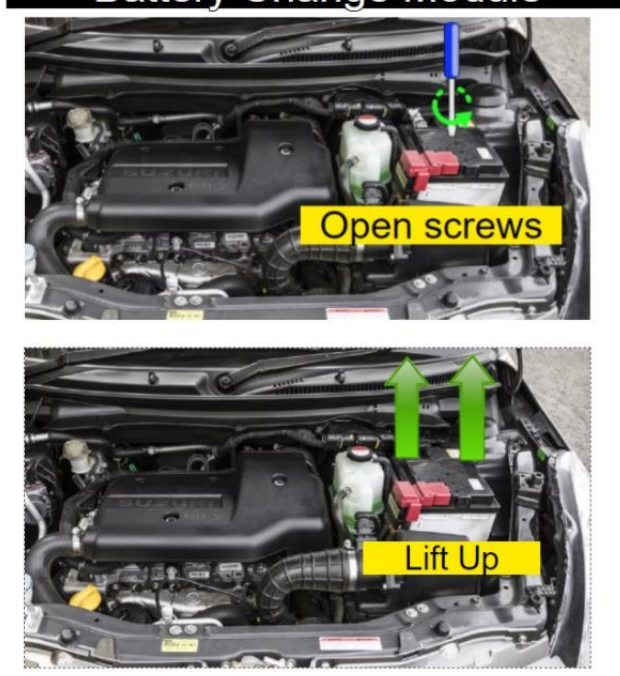

Fig. 1. Demonstrating the change of battery.

We also made a real-time screen marker, using which the user just needs to place his phone camera in front of car, and it will display the user's screen view to an expert in the company, and he just needs to draw from his fingers on the screen to guide the user for troubleshooting a problem, and it will display results in user's screen. But as the current stage of development is considered, we have made a marker drawing on screen while camera display is rendered. Only we need a server to make two screens share display. We have made an
AR guide by animating the $3 \mathrm{~d}$ objects and added an audio reference also for better understanding. For now we just added a recorded voice, but in later phase we will use Cloud Text To Speech Service, to reduce the app size. Google AR Core SDK's are also available, but it requires high performance phones (¿API 24) which is usually not affordable by everyone. But our application is supported on even $1 \mathrm{gb}$ ram phones having Android 5.0 (API 21).

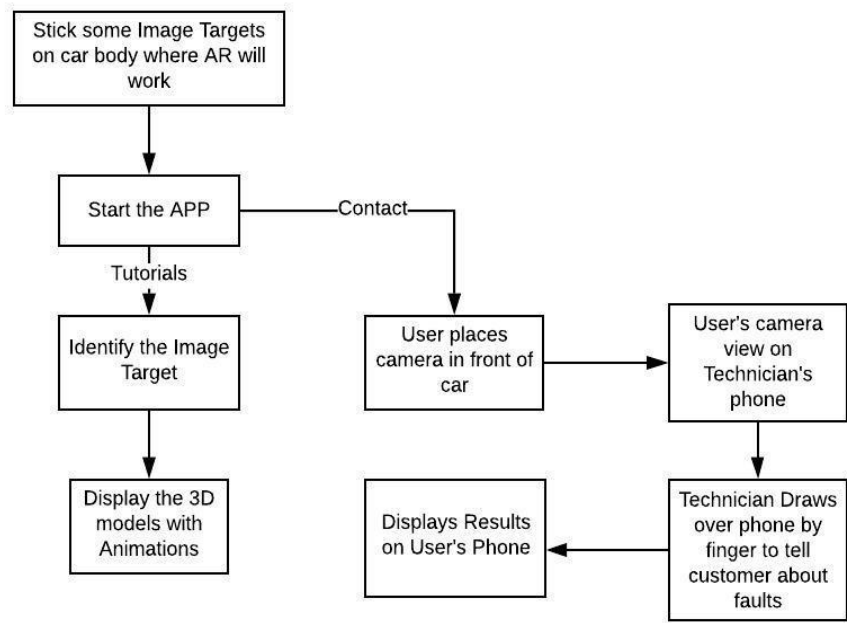

Fig. 2. Application workflow diagram.

\section{ARCHITECTURE}

We used Blender software to export obj files and imported them in Unity3D. We didn't used Scene form plugin of Android, as AR CORE is not supported on many devices, so our app can get more users as it is supported on most of the phones out there. We repeated the use of 3D models in scenes wherever possible because using different 3D models for different scenes can consume more storage and devices resources to render, but we can't ignore the fact that user gets bored by seeing the same stuff repeatedly. So we used 3D models that were not colored, and inside the different scenes, we just changed the materials prefabs, so that in different scenes the same 3D model will be displayed but in different colors. We used a C\# script to draw over the AR Camera, it using Ray-Casting technique to draw over the screen, on an invisible plane object. We have use Image Targets as some unique stickers that we will place over car where AR is to be displayed. As the camera recognizes those Image Targets (stickers), it will start displaying the 3D models inside it. Full vehicle part detection is not easy on low specs devices, so we will use Image Recognition of targets. We also tried to make this AR from scratch, so we used Computer Vision tool in Python called OpenCV, but its performance was not meeting to deploy as a real-life application. There are some 


\section{International Journal of Engineering Applied Sciences and Technology, 2020 \\ Vol. 5, Issue 7, ISSN No. 2455-2143, Pages 262-264 \\ Published Online November 2020 in IJEAST (http://www.ijeast.com)}

paid tools available for development of AR softwares like Microsoft Hololens, Amazon Sumerian etc, through which we can also detect the exact hardware then display our AR animation, but due to limited resources we are using Image Targets for now We have built a chatbot module that understands the problem faced by the user and tells the respective solution. It the problem can be fix by the user himself, the application will respond the solution, or it will ask the user to go to a specialist technician. It includes responses for almost all the warnings such as smoke, strange noise from car, key related problems etc. You can either write your question or simply hold down the button to speak you question.

\section{CONCLUSION}

We made an Augmented Reality Application for smartphones to guide people in ordinary maintenance of their car anytime, anywhere. For the next phase we are going to use web scrapping technique in C\#, Scrapy Sharp, that will process the query in real-time and fetches the results from internet and hence user gets more appropriate solution for his query. We also need an intermediate server that will allow two screens to render each other's display in real-time as a screen sharing. software eg: AnyDesk. Only issue that is in our solution is lack of access to Cloud services. But this time we are arranging for a premium Cloud Service to complete the lacking part of our solution. Further updates more tutorials can be added to the application. The aim is to provide minimal use of human efforts and making things done by automation.

\section{REFERENCE}

[1] Henderson, S. J., and Feiner, S. K. (2007). Augmented reality for maintenance and repair (armar). Columbia Univ New York Dept of Computer Science.

[2] Friedrich, W., Jahn, D., and Schmidt, L. (2002, September). ARVIKAAugmented Reality for Development, Production and Service. In ISMAR (Vol. 2002, pp. 3-4)

[3] de Almeida, P. B. M. C. Management of Automotive Maintenance Components..

[4] Januszka Marcin and Panfil Wawrzyniec, Augmented reality techniques for vehicle maintenance,Silesian University of Technology.

[5] Lombard, M., and Ditton, T. (1997). At the heart of it all: the concept of presence. J. Comput. Mediat. Commun. 3.

[6] Alexander, T., Westhoven, M., and Conradi, J. (2017). "Virtual environments for competency-oriented education and training," in Advances in Human Factors, Business Management, Training and Education, (Berlin: Springer International Publishing), 23-29
[7] Andersen, S. M., and Thorpe, J. S. (2009). An if-thEN theory of personality: significant others and the relational self. J. Res. Pers. 43, 163-170

[8] Azevedo, R. T., Bennett, N., Bilicki, A., Hooper, J., Markopoulou, F., and Tsakiris, M. (2017). The calming effect of a new wearable device during the anticipation of public speech. Sci. Rep. 7:2285.

[9] Azuma, R., Baillot, Y., Behringer, R., Feiner, S., Julier, S., and MacIntyre, B. (2001). Recent advances in augmented reality. IEEE Comp. Graph. Appl. 21, 34-47.

[10] Bacca, J., Baldiris, S., Fabregat, R., and Graf, S. (2014). Augmented reality trends in education: a systematic review of research and applications. J. Educ. Technol. Soc. $17,133$.

[11] Bailenson, J. N., Yee, N., Merget, D., and Schroeder, R. (2006). The effect of behavioral realism and form realism of real-time avatar faces on verbal disclosure, nonverbal disclosure, emotion recognition, and copresence in dyadic interaction. Presence 15, 359-372.

[12] Baños, R. M., Botella, C., Garcia-Palacios, A., Villa, H., Perpiñá, C., and Alcaniz, M. (2000). Presence and reality judgment in virtual environments: a unitary construct.

[13] Biocca, F., Harms, C., and Gregg, J. (2001). "The networked minds measure of social presence: pilot test of the factor structure and concurrent validity," in 4th Annual International Workshop on Presence, Philadelphia, PA, 1-9.

[14] Bohil, C. J., Alicea, B., and Biocca, F. A. (2011). Virtual reality in neuroscience research and therapy. Nat. Rev. Neurosci.

[15] Burdea, G., Richard, P., and Coiffet, P. (1996). Multimodal virtual reality: input-output devices, system integration, and human factors. Int. J. Hum. Compu. Interact. 